\title{
Opportunistic diseases among HIV-infected patients: a multicenter-nationwide Korean HIV/ AIDS cohort study, 2006 to 2013
}

Youn Jeong Kim ${ }^{1}$, Jun Hee Woo², Min Ja Kim³, Dae Won Park ${ }^{3}$, Joon-Young Song Shin Woo Kim ${ }^{4}$, Jun Yong Choi ${ }^{5}$, June Myung Kim5, Sang Hoon Han ${ }^{5}$, Jin-Soo Lee ${ }^{6}$, Bo Youl Choi ${ }^{7}$, Joo Shil Lee ${ }^{8}$, Sung-Soon Kim ${ }^{8}$, Mee-Kyung Kee ${ }^{8}$, Moon Won Kang ${ }^{1}$, and Sang Il Kim ${ }^{1}$

${ }^{1}$ Division of Infectious Disease, Department of Internal Medicine, College of Medicine, Seoul St. Mary's Hospital, The Catholic University of Korea, Seoul; ${ }^{2}$ Department of Infectious Disease, Asan Medical Center, University of Ulsan College of Medicine, Seoul; ${ }^{3}$ Department of Internal Medicine, Korea University College of Medicine, Seoul; ${ }^{4}$ Department of Internal Medicine, Kyungpook National University School of Medicine, Daegu; ${ }^{5}$ Department of Internal Medicine, Yonsei University College of Medicine, Seoul; ${ }^{6}$ Department of Internal Medicine, Inha University School of Medicine, Incheon; ${ }^{7}$ Department of Preventive Medicine, Hanyang University College of Medicine, Seoul; ${ }^{8}$ Korea Centers for Disease Control and Prevention, Cheongju, Korea

Received: October 22, 2014 Revised : January 6, 2015 Accepted: April 27, 2015

\section{Correspondence to}

Sang Il Kim, M.D.

Division of Infectious Disease, Department of Internal Medicine, College of Medicine, Seoul St. Mary's Hospital, The Catholic University of Korea, 222 Banpo-daero, Seocho-gu, Seoul 06591, Korea

Tel: $+82-2-2258-1240$

Fax: +82-2-2258-1254

E-mail: drksi@catholic.ac.kr
Background/Aims: The frequencies of opportunistic diseases (ODs) vary across countries based on genetic, environmental, and social differences. The Korean HIV/AIDS cohort study was initiated in 2006 to promote research on human immunodeficiency virus (HIV) infection in Korea, and to provide a logistical network to support multicenter projects on epidemiological, clinical, and laboratory aspects of HIV infection. This study evaluated the prevalence of ODs among HIV-infected patients in the era of highly active antiretroviral therapy, and the risk factors associated with ODs.

Methods: The study enrolled 1,086 HIV-infected patients from 19 hospitals. This study examined the baseline data of the HIV/AIDS Korean cohort study at the time of enrollment from December 2006 to July 2013.

Results: Candidiasis was the most prevalent opportunistic infection $(\mathrm{n}=176$, 16.2\%), followed by Mycobacterium tuberculosis infection ( $\mathrm{n}=120,10.9 \%)$, Pneumocystis jirovecii pneumonia $(\mathrm{n}=121,11.0 \%)$, cytomegalovirus infection $(\mathrm{n}=52,4.7 \%)$, and herpes zoster $(n=44,4.0 \%)$. The prevalence rates of Kaposi's sarcoma $(n=8$, $0.7 \%)$ and toxoplasmosis $(n=4,0.4 \%)$ were very low compared with other countries. The risk factors for ODs were a low $\mathrm{CD}_{4} \mathrm{~T}$ cell count at the time of HIV diagnosis (odds ratio [OR], 1.01; $p<0.01$ ), current smoking (OR, 2.27; $p=0.01$ ), current alcohol use (OR, 2.57; $p=0.04)$, and a history of tuberculosis (OR, 5.23; $p<0.01$ ). Conclusions: Using recent Korean nationwide data, this study demonstrated that an important predictor of ODs was a low $\mathrm{CD}_{4} \mathrm{~T}$ cell count at the time of HIV diagnosis. Tuberculosis remains one of the most important ODs in HIV-infected patients in Korea.

Keywords: AIDS-related opportunistic infections; HIV; Korea; Tuberculosis 


\section{INTRODUCTION}

Globally, an estimated 34 million people were infected with human immunodeficiency (HIV) at the end of 2011. In Korea, after the first case of HIV infection was reported in 1985 , the number of cumulative HIV survivors rose to approximately 7,000 at the end of 2011, although the overall prevalence in the population was very low $(<1 \%)$ [1]. HIV infection leads to immunosuppression, which can result in life-threatening opportunistic diseases (ODs). Combination antiretroviral therapy reduced the mortality and morbidity [2,3], but despite advances in antiretroviral therapy, ODs remain one of the main causes of morbidity and mortality in HIV/AIDS patients.

The frequencies of ODs may vary across countries because of differences in genetic, environmental, and social backgrounds. Two studies have examined the epidemiology and clinical manifestations of ODs in Korean patients with HIV/AIDS $[4,5]$. The introduction of combination antiretroviral therapy and the use of prophylactic antimicrobials for ODs have changed the prevalence of ODs over time. Consequently, an understanding of the current epidemiology of ODs among HIV/ AIDS patients should help to establish guidelines for the prevention of ODs. This study evaluated the prevalence of ODs among HIV-infected patients in the era of highly active antiretroviral therapy, and the risk factors associated with ODs.

\section{METHODS}

\section{Study design and time period}

The cohort recruited patients older than 18 years infected with HIV after obtaining informed consent. Trained researchers from all centers prospectively collected information every 6 months, using a standardized protocol. The information included the medical history, socioeconomic status, physical findings, laboratory findings including immunological and virological status, and ODs. In this study, we determined the prevalence of ODs using the baseline data of the HIV/AIDS cohort study at the time of enrollment, from December 2006 to July 2013.

\section{Definition}

ODs were diagnosed from the clinical, laboratory, and pathology findings, following published criteria [6]. A diagnosis of tuberculosis (TB) was based on compatible symptoms/signs and positive acid-fast bacilli (AFB) smear, culture, polymerase chain reaction (PCR), and histological or radiological findings compatible with TB. Disseminated TB was defined when more than two sites were involved [7]. Pneumocystis jirovecii pneumonia was confirmed based on compatible symptoms/signs, and radiological findings such as bilateral, diffuse interstitial infiltrates, demonstration of the cyst wall on methenamine silver staining, or a positive P. jirovecii PCR. Cytomegalovirus (CMV) viremia was detected using a CMV PCR assay. CMV retinitis was diagnosed by an ophthalmologist. CMV disease such as pneumonia or hepatitis was diagnosed based on compatible symptoms and pathology staining for CMV.

\section{Ethics approval}

Ethics approval was obtained from all participating hospitals.

\section{Data analysis}

Data are provided as mean \pm standard deviation, or as proportions. Student $t$ test or the Mann-Whitney $U$ test was used to analyze continuous variables, and the chisquare test or Fisher exact test was used for categorical variables. We analyzed the risk factors associated with developing ODs in HIV-infected patients using both univariate and multivariate logistic regression analyses. Variables with a $p$ value $<0.1$ in the univariate analysis were added in a forward stepwise manner and selected to create the final model for multivariate analysis with forward variables. Data were analyzed using the SPSS version 16.0 (SPSS Inc., Chicago, IL, USA), and a $p<0.05$ was considered statistically significant.

\section{RESULTS}

The study enrolled 1,086 HIV-infected patients (1,007 males and 79 females; median age, 41 years) at 19 university hospitals from 2006 to 2012 (Table 1). The majority of patients $(n=601,55.4 \%)$ were 30 to 50 years of age. The median $\mathrm{CD}_{4} \mathrm{~T}$ cell count at enrollment was 352 cells/ 
Table 1. Comparisons of demographic characteristics between patients with ODs and without ODs

\begin{tabular}{|c|c|c|c|c|}
\hline Characteristic & Total $(\mathrm{n}=1,086)$ & ODs $(+)(n=297)$ & $\operatorname{ODs}(-)(\mathrm{n}=789)$ & $p$ value \\
\hline Age, yr & $41(33-50)$ & $44(36-52)$ & $41(32-50)$ & $<0.01$ \\
\hline Male sex & $1,007(92.7)$ & $279(93.9)$ & $728(92.3)$ & 0.37 \\
\hline \multicolumn{5}{|l|}{ Transmission route } \\
\hline Sexual contact & $945(97.0)^{a}$ & $261(87.8)^{\mathrm{a}}$ & $684(86.7)^{\mathrm{a}}$ & 0.89 \\
\hline Transfusion or blood product & $24(2.5)^{\mathrm{a}}$ & $9(3.0)^{\mathrm{a}}$ & $15(1.9)^{\mathrm{a}}$ & 0.87 \\
\hline Injection drug use & $5(0.5)^{\mathrm{a}}$ & $3(1.0)^{\mathrm{a}}$ & $2(0.3)^{\mathrm{a}}$ & 0.09 \\
\hline Vertical transmission & o & o & o & - \\
\hline $\mathrm{CD}_{4} \mathrm{~T}$ cell count at enrollment, cell $/ \mathrm{mm}^{3}$ & $352(207-514)$ & $240(111-395)$ & $388(254-560)$ & $<0.01$ \\
\hline $\mathrm{CD}_{4} \mathrm{~T}$ cell at HIV diagnosis, cell $/ \mathrm{mm}^{3}$ & $237(93-377)$ & $75(25-203)$ & $279 \cdot 5(169-420)$ & $<0.01$ \\
\hline Viral load at enrollment, log copies/mL & $5.69(3.88-6.24)$ & $5.04(4.17-5.56)$ & $4.57(3.8-6.09)$ & 0.62 \\
\hline HARRT at enrollment & $730(67 \cdot 3)$ & $232(78.1)$ & $498(63.1)$ & $<0.01$ \\
\hline Body mass index, $\mathrm{kg} / \mathrm{m}^{2}$ & $21.5(19.4-23.5)$ & $21.1(19-23.5)$ & $21.6(19.8-23.6)$ & 0.77 \\
\hline \multicolumn{5}{|l|}{ Smoking } \\
\hline Current & $465(71.5)^{\mathrm{a}}$ & $111(59.4)$ & $354(76.5)$ & $<0.01$ \\
\hline Former & $648(68.0)^{\mathrm{a}}$ & $189(70.0)$ & $459(67.2)$ & 0.40 \\
\hline \multicolumn{5}{|l|}{ Alcohol } \\
\hline Current & $483(73.0)^{\mathrm{a}}$ & $106(57.9)$ & $377(78.7)$ & $<0.01$ \\
\hline Former & $656(70.0)^{a}$ & $183(68.3)$ & $473(70.0)$ & 0.47 \\
\hline Tuberculosis history & $196(18.0)$ & $115(38.7)$ & $81(10.2)$ & $<0.01$ \\
\hline Hypertension & $112(10.3)$ & $29(9.7)$ & $83(10.5)$ & 0.84 \\
\hline Diabetes mellitus & $71(6.5)$ & $17(5 \cdot 7)$ & $54(6.8)$ & 0.38 \\
\hline Previous BCG history & $377(54.5)^{\mathrm{a}}$ & $108(36.4)$ & $269(34.1)$ & 0.71 \\
\hline
\end{tabular}

Values are presented as median (interquartile range) or number (\%).

OD, opportunistic disease; HIV, human immunodeficiency; HARRT, highly active antiretroviral therapy; BCG, Bacillus Calmette-Guérin.

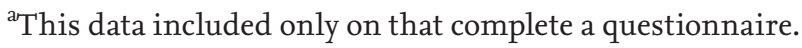

$\mathrm{mm}^{3}$ (interquartile range [IQR], 207 to 514 ), and $22.9 \%$ of the patients $(n=206)$ had fewer than 200 cells $/ \mathrm{mm}^{3}$. The median viral load at enrollment was $5.69 \log$ copies $/ \mathrm{mL}$ (IQR, 3.88 to 6.24 ), while $53.2 \%$ of the patients had undetectable viral loads. At enrollment, $76 \%$ of the patients (n =355) were receiving antiretroviral agents (Table 1 ).

\section{Opportunistic diseases}

Among the 1,086 HIV-infected patients, 613 ODs developed in 297 patients (24.4\%), a rate of 2.1 episodes/patient. Table 2 summarizes the ODs seen in our study. Candidiasis was the most prevalent OD $(n=176,16.2 \%)$, and was mainly oral candidiasis $(n=120)$, followed by esophageal candidiasis $(n=54)$. TB was the second most frequent $\mathrm{OD}(\mathrm{n}=120,11.0 \%)$. Among the patients with
TB, 28.3\% ( $\mathrm{n}=34)$ were taking antiretroviral treatment and $54.2 \%(n=65), 15.8 \%(n=19)$, and $15 \%(n=18)$ had pulmonary, lymph node, and disseminated TB, respectively. The main diagnostic method for TB was the AFB smear $(\mathrm{n}=58,48.3 \%)$, followed by AFB culture $(\mathrm{n}=49$, $49 \%), \operatorname{PCR}(n=28,23.3 \%)$, and pathology $(n=20,16.6 \%)$. Based on clinical and radiological characteristics, anti-TB medication was given empirically to three patients with negative results on a routine test for TB. Of the 19 patients who had antimicrobial drug resistance tests, $26.3 \%(\mathrm{n}=5)$ were resistant to isoniazid, $15.8 \%(\mathrm{n}=$ 3) to rifampin, and $10.5 \%(n=2)$ to ethambutol. P.jirovecii pneumonia $(\mathrm{n}=121,11.1 \%)$ was the third most common OD, followed by CMV infection ( $\mathrm{n}=52,4.7 \%$ ), and herpes zoster $(n=44,4.0 \%)$. CMV retinitis was found in 19 
Table 2. Distribution of opportunistic disease and $\mathrm{CD}_{4} \mathrm{~T}$ cell count

\begin{tabular}{|c|c|c|}
\hline Variable & No. of cases & $\mathrm{CD}_{4} \mathrm{~T}$ cell, cell $/ \mathrm{mm}^{3}$ \\
\hline Candidiasis & $176(16.2)$ & \\
\hline Oral candidiasis & 120 & $49(17-133)$ \\
\hline Esophageal candidiasis & 54 & $43(19-95 \cdot 5)$ \\
\hline Respiratory candidiasis & 2 & - \\
\hline Tuberculosis & $120(11.0)$ & $87(37-205)$ \\
\hline Nontuberculous Mycobacteria infection & $2(0.2)$ & - \\
\hline Pneumocystis jirovecii pneumonia & $121(11.1)$ & $30(11-68)$ \\
\hline CMV infection & $52(4 \cdot 7)$ & $25.5\left(12.75^{-101.5)}\right.$ \\
\hline Retinitis & 19 & \\
\hline Pneumonia & 13 & \\
\hline Hepatitis & 9 & \\
\hline Colitis & 2 & \\
\hline Cytomegalovirus viremia & 9 & \\
\hline Herpes zoster & $44(4.0)$ & $185(116.5-273.5)$ \\
\hline Herpes simplex virus infection & $8(0.7)$ & $128(10-320)$ \\
\hline Recurrent pneumonia & $7(0.7)$ & $62.5(12-170)$ \\
\hline Progressive multifocal leukoencephalopathy & $6(0.6)$ & $107(64.25-154)$ \\
\hline Cryptococcosis & $6(0.6)$ & $31.2(9 \cdot 5-76.75)$ \\
\hline Toxoplasmosis & $4(0.4)$ & $107(25 \cdot 5-246.25)$ \\
\hline Isosporiasis & $1(0.09)$ & - \\
\hline Non-Hodgkin's lymphoma & $4(0.4)$ & $128(26-222.5)$ \\
\hline Burkitt lymphoma & 1 & \\
\hline Diffuse large B-cell lymphoma & 3 & \\
\hline Kaposi’s sarcoma & $8(0.7)$ & $133(28-269)$ \\
\hline Cervical carcinoma & $1(0.09)$ & - \\
\hline
\end{tabular}

Values are presented as number (\%) or median (interquartile range).

patients. The prevalence rates of Kaposi's sarcoma $(\mathrm{n}=8$, $0.7 \%)$ and toxoplasmosis $(\mathrm{n}=4,0.4 \%)$ were low.

The median $\mathrm{CD}_{4} \mathrm{~T}$ cell count was 49 cells $/ \mathrm{mm}^{3}$ (IQR, 17 to 133) in patients with esophageal candidiasis, 30 cells $/ \mathrm{mm}^{3}$ (IQR, 11 to 68 ) in P. jirovecii pneumonia cases, and 87 cells $/ \mathrm{mm}^{3}$ (IQR, 37 to 205) in TB cases (Table 2). When patients were first diagnosed with HIV, $13.9 \%$ $(n=151)$ had AIDS-defining illnesses. The most prevalent indicator of AIDS was P. jirovecii pneumonia ( $\mathrm{n}=91$, 60.3\%), followed by Mycobacterium tuberculosis infection $(\mathrm{n}=69,45.6 \%)$ and esophageal candidiasis $(\mathrm{n}=38,25.2 \%)$.

Fig. 1 shows the distribution of the $\mathrm{CD}_{4}$ cell count in study patients with OD. Patients who had a CD4 T cell count less than 50 cells $/ \mathrm{mm}^{3}$ had a high prevalence of P. jirovecii pneumonia (66.9\%), CMV infection (56.2\%), or esophageal or respiratory candidiasis (56.3\%). A high prevalence of TB (73.8\%) was found in patients with a $\mathrm{CD}_{4} \mathrm{~T}$ cell count less than 200 cells $/ \mathrm{mm}^{3}$.

\section{Risk factors for opportunistic diseases}

Table 1 compares the demographic characteristics of patients with and without ODs. There were no differences in sex, transmission route, viral load, or body mass index. Patients with ODs were significantly older than patients without ODs (median, 44; IQR, 36 to 52 years vs. median, 41 ; IQR, 32 to 50 years; $p<0.01$ ), and had a lower $\mathrm{CD}_{4} \mathrm{~T}$ cell count at the time of HIV diagnosis (median, 75; IQR, 25 to 203 cells $/ \mathrm{mm}^{3}$ vs. median, 279.5; IQR, 169 to 420 cells $/ \mathrm{mm}^{3}, p<0.01$ ), and also had a lower $\mathrm{CD}_{4} \mathrm{~T}$ cell count at enrollment (median, 240; IQR, 111 to 395 cells/ 


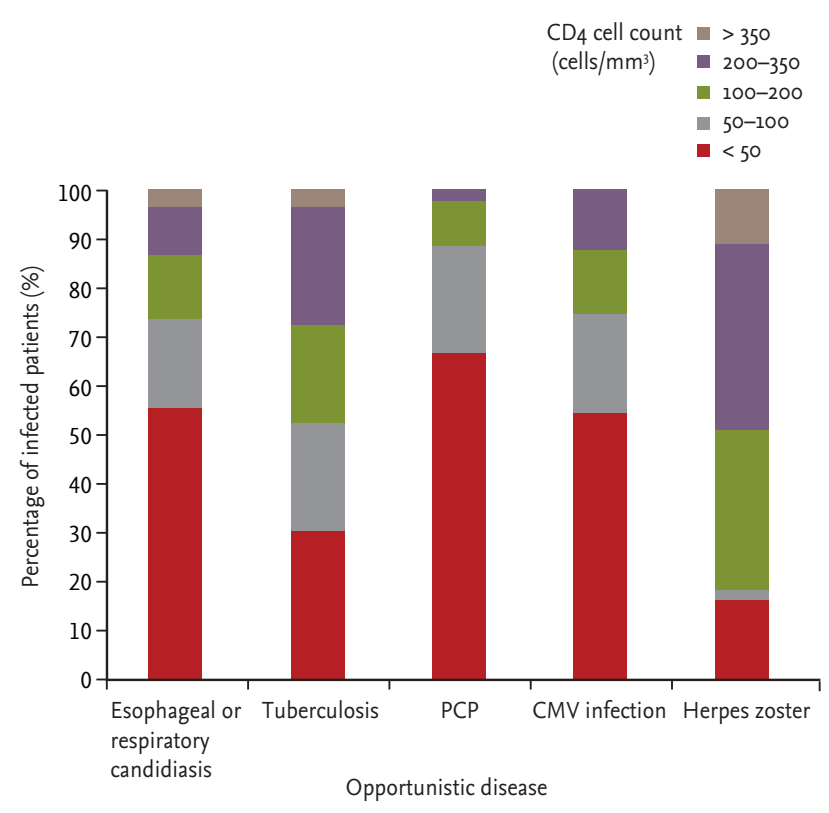

Figure 1. Distribution of $\mathrm{CD}_{4}$ cell count in study patients with opportunistic disease. PCP, Pneumocystis carinii pneumonia; CMV, cytomegalovirus.

$\mathrm{mm}^{3}$ vs. median, 388 ; IQR, 254 to 560 cells $/ \mathrm{mm}^{3}, \mathrm{p}<0.01$ ). The patients with ODs were more likely to be current smokers $(p<0.01)$, consume alcohol $(p<0.01)$, and have prior TB $(p<0.01)$ than those without ODs.

In the univariate analysis, older age (OR, $0.98 ; p<0.01)$, a lower $\mathrm{CD}_{4} \mathrm{~T}$ cell count at the time of HIV diagnosis (OR, 1.01; $p<0.01)$, a lower $\mathrm{CD}_{4} \mathrm{~T}$ cell count at cohort study enrollment (OR, 1.01; $p<0.01)$, antiretroviral therapy (OR, 2.47; $p<0.01)$, current smoking (OR, 2.22; $p=$ 0.001), current alcohol consumption (OR, 2.68; $p<0.01)$, and a history of TB treatment (OR, 5.61; $p<0.01)$ were associated with ODs among HIV-infected patients (Table 3). The multivariate analysis showed that a low $\mathrm{CD}_{4}$ T cell count at the time of HIV diagnosis (OR, 1.01; $p<$ o.01), current smoking (OR, 2.27; $p=0.01)$, current alcohol consumption (OR, 2.57; $p=0.04)$, and a history of TB $(\mathrm{OR}, 5.23 ; p<0.01)$ were independent risk factors for ODs.

\section{Outcomes}

During the follow-up period, 26 patients $(2.4 \%)$ died. The ODs-related mortality was $0.7 \%(n=8)$, accounting for $33.3 \%$ of deaths. The causes of OD-related death were pneumonia of unknown origin $(n=4), T B(n=1)$, P. jirovecii pneumonia $(\mathrm{n}=1)$, and P. jirovecii pneumonia combined with TB $(n=1)$.

\section{DISCUSSION}

Before the introduction of combination antiretroviral therapy, ODs were the main cause of death in HIV-infected patients. However, advances in antiviral treatment led to a dramatic decline in ODs $[2,8,9]$. In our study, although $24.4 \%$ of the HIV-infected patients developed ODs, they were not the main cause of death, unlike previous studies.

In our series, the most prevalent OD was candidiasis, followed by TB and P. jirovecii pneumonia. In a study of 173 HIV-infected patients performed from 1985 to 1998 , TB was the most frequent opportunistic infection (25\% of patients), followed by candidiasis and herpes zoster

Table 3. Risk factors for opportunistic disease among HIV patients

\begin{tabular}{|c|c|c|c|c|c|c|}
\hline \multirow{2}{*}{ Factor } & \multicolumn{3}{|c|}{ Univariate analysis } & \multicolumn{3}{|c|}{ Multivariate analysis } \\
\hline & OR & $95 \% \mathrm{CI}$ & $p$ value & OR & $95 \% \mathrm{CI}$ & $p$ value \\
\hline Age & 0.98 & $0.96-0.99$ & $<0.01$ & 0.98 & $0.95^{-1.01}$ & 0.29 \\
\hline Body mass index & 1.01 & $0.98-1.02$ & 0.77 & & & \\
\hline $\mathrm{CD}_{4} \mathrm{~T}$ cell, HIV diagnosis & 1.01 & $1.01-1.05$ & $<0.01$ & 1.01 & $1.03-1.07$ & $<0.01$ \\
\hline Viral load at enrollment & 1 & $0.90-1.02$ & 0.67 & & & \\
\hline $\mathrm{CD}_{4} \mathrm{~T}$ cell at enrollment & 1.01 & $1.02-1.04$ & $<0.01$ & 1.001 & $1.01-1.03$ & 0.45 \\
\hline HAART at enrollment & 2.47 & $1.79-3.41$ & $<0.01$ & 1.41 & $0.55-3.56$ & 0.47 \\
\hline Current smoking & 2.22 & $1.55-3.19$ & $<0.01$ & 2.27 & $1.22-4.26$ & 0.01 \\
\hline Current alcohol & 2.68 & $1.86-3.87$ & $<0.01$ & 2.57 & $1.34-4.91$ & 0.04 \\
\hline Tuberculosis history & 5.61 & $4.03-7.7$ & $<0.01$ & 5.23 & $2.69-10.2$ & $<0.01$ \\
\hline
\end{tabular}

HIV, human immunodeficiency; OR, odds ratio; CI, confidence interval; HAART, highly active antiretroviral therapy. 
[5]. In another study of $176 \mathrm{HIV}$-infected patients from 1985 to 2000 , candidiasis was the most prevalent OD, followed by P. jirovecii pneumonia and TB [4]. Based on our data, TB can be considered a re-emerging OD in the HIV population in Korea. The prevalence of ODs in HIV patients varies by location and is $32.5 \%$ in China and 27.4\% in Ethiopia and New York city [10-12]. In developing countries, TB remains the most common cause of death among HIV-infected patients, similar to the situation in Korea, and unlike countries with low risks of TB $[13,14]$. In Korea, the estimated TB incidence in 2012 was 108 per 100,000 population, the highest among Organization for Economic Cooperation and Development countries. The identification and treatment of latent TB infection has lowered the incidence of TB in developed countries. The current guidelines for the management of TB in Korea recommend treating latent TB in HIV-infected patients [7]. Until now, many doctors in Korea have tended to avoid giving TB treatment for latent TB for several reasons: toxicity, drug interactions, and concern about drug resistance. In addition, our data showed that TB developed in patients with a low $\mathrm{CD}_{4}$ $\mathrm{T}$ cell count, and many studies demonstrated that impaired cellular immunity was associated with TB reactivation $[15,16]$. After the guidelines for latent TB are applied to clinical practice in Korea, further studies must examine the effect on the incidence of TB. In our series, a history of TB was associated with ODs among HIV-infected patients. Several studies have also demonstrated that a history of TB was usually associated with TB infection $[17,18]$. In intermediate TB endemic areas, TB reactivation is more common in HIV-infected patients than de novo TB. There were two cases of nontuberculous Mycobacteria infection in our study. Our study used the patient data at the time of enrollment, and further studies will examine prospectively collected cohort data.

Our study found a low prevalence of Kaposi's sarcoma and toxoplasmosis compared with other countries. This is because of the geographic variation in Kaposi's sarcoma. de Sanjose et al. [19] reported that the prevalence of Kaposi's sarcoma-associated herpesvirus in the general female population in Korea was only 4.93\%.

The associated risk factors for ODs in our study were a low $\mathrm{CD}_{4} \mathrm{~T}$ cell count at the time of HIV diagnosis, smoking, alcohol consumption, and a history of TB. A low $\mathrm{CD}_{4} \mathrm{~T}$ cell count is associated with the develop- ment of ODs $[10,20]$. A study of homeless HIV/AIDS patients in Korea showed that they are at an advanced stage at admission, which leads to ODs and an unfavorable outcome [21]. Our results showed that $97 \%$ of HIV-infected patients with $P$. jirovecii pneumonia had CD4 T cell counts less than 200 cells $/ \mathrm{mm}^{3}$; this supports recent guidelines that patients with such low $\mathrm{CD}_{4}+\mathrm{T}$ cell counts should receive prophylaxis for $P$. jirovecii. Smoking could influence inflammatory and immune responses in the oral cavity, and smoking was associated with oral candidiasis in some studies [22,23].

This study had several limitations. First, we included only patient data at study enrollment; prospective longitudinal data will provide more accurate data on OD incidence and change in ODs in Korea. Second, we did not calculate the risk factors for ODs for each disease, and did not describe the clinical course of each OD. Third, we included only the data from patients in our cohort study. However, this study enrolled patients from multiple centers that covered most areas of Korea, so it is likely to be representative epidemiological data for Korea.

In conclusion, there was a range of ODs among HIV-infected patients in Korea, the most prevalent being candidiasis, $M$. tuberculosis infection, $P$. jirovecii pneumonia, CMV infection, and herpes zoster, while toxoplasmosis and HIV-related malignancy, such as Kaposi's sarcoma, were rare. A major predictor of OD was a low $\mathrm{CD}_{4} \mathrm{~T}$ cell count at the time of HIV diagnosis. Based on our data, strategies to diagnose HIV at an early stage of infection, and timely antiretroviral therapy, are needed to reduce ODs among HIV-infected patients in Korea.

\section{KEY MESSAGE}

1. Candidiasis, Mycobacterium tuberculosis infection, Pneumocystis jirovecii pneumonia, cytomegalovirus infection, and herpes zoster were common opportunistic diseases among Korean human immunodeficiency virus (HIV)-infected patients.

2. Toxoplasmosis and HIV-related malignancy, such as lymphoma, Kaposi's sarcoma, and cervical carcinoma, were rare in Korea.

3. A major predictor of opportunistic diseases was a low $\mathrm{CD}_{4} \mathrm{~T}$ cell count at the time of HIV diagnosis. 


\section{Conflict of interest}

No potential conflict of interest relevant to this article was reported.

\section{Acknowledgments}

This study was supported by a fund of the Chronic Infectious Disease Cohort Study (Korea HIV/AIDS Cohort Study [grant number: 4800-4859-304-260, 2013-E5100602]) from the Korea Centers for Disease Control and Prevention. We thank the members of the HIV Korean cohort. The Korea HIV/AIDS cohort study group consists of Moon Won Kang, Min Ja Kim, Jun Hee Woo, Sang Il Kim, Youn Jeong Kim, Dae Won Park, Won Suk Choi, Jang Wook Sohn, Seong Han Kim, Seong-Heon Wie, Ji-An Hur, Yeon Joon Park, Shin-Woo Kim, HyunHa Chang, Yoo Joo Kim, Joon Young Song, Joong Shik Eom, Jin Seo Lee, Jacob Lee, Hye Won Jeong, Jin Soo Lee, Hee Jung Choi, Seung Soon Lee, June Myung Kim, Jun Yong Choi, Sang Hoon Han, Nam Su Ku, Jin Young Ahn, Hyo-Youl Kim, Young Keun Kim, Yong Kyun Cho, Yoon Soo Park, Seung Kwan Lim, Young Hwa Choi, Choi Bo Youl, Hee Suk Park, Mee-Kyng Kee, Joo Shil Lee, and Sung Soon Kim.

\section{REFERENCES}

1. World Health Organization. Global health observatory data repository [Internet]. Geneva: World Health Organization, c2015 [cited 2015 Sep 22]. Available from: http:// apps.who.int/ghodata.

2. Buchacz K, Baker RK, Palella FJ Jr, et al. AIDS-defining opportunistic illnesses in US patients, 1994-2007: a cohort study. AIDS 2010;24:1549-1559.

3. Schwarcz L, Chen MJ, Vittinghoff E, Hsu L, Schwarcz S. Declining incidence of AIDS-defining opportunistic illnesses: results from 16 years of population-based AIDS surveillance. AIDS 2013;27:597-605.

4. Kim JM, Cho GJ, Hong SK, et al. Epidemiology and clinical features of HIV infection/AIDS in Korea. Yonsei Med J 2003;44:363-370.

5. Oh MD, Park SW, Kim HB, et al. Spectrum of opportunistic infections and malignancies in patients with human immunodeficiency virus infection in South Korea. Clin Infect Dis 1999;29:1524-1528.

6. The Korean Society for AIDS. Clinical guidelines for the treatment and prevention of opportunistic infections in HIV infected Koreans. Infect Chemother 2012;44:93-139.

7. Joint Committee for the Development of Korean Guideline for Tuberculosis. Korean guidelines for tuberculosis. 1st ed. Cheongju: Korea Centers for Disease Control and Prevention, 2011.

8. Seage GR 3rd, Losina E, Goldie SJ, Paltiel AD, Kimmel AD, Freedberg KA. The relationship of preventable opportunistic infections, HIV-1 RNA, and $\mathrm{CD}_{4}$ cell counts to chronic mortality. J Acquir Immune Defic Syndr 2002;30:421-428.

9. Egger M, Hirschel B, Francioli P, et al. Impact of new antiretroviral combination therapies in HIV infected patients in Switzerland: prospective multicentre study. Swiss HIV Cohort Study. BMJ 1997;315:1194-1199.

10. Damtie D, Yismaw G, Woldeyohannes D, Anagaw B. Common opportunistic infections and their $\mathrm{CD}_{4}$ cell correlates among HIV-infected patients attending at antiretroviral therapy clinic of Gondar University Hospital, Northwest Ethiopia. BMC Res Notes 2013;6:534.

11. Xiao J, Gao G, Li Y, et al. Spectrums of opportunistic infections and malignancies in HIV-infected patients in tertiary care hospital, China. PLoS One 2013;8:e75915.

12. Hanna DB, Gupta LS, Jones LE, Thompson DM, Kellerman SE, Sackoff JE. AIDS-defining opportunistic illnesses in the HAART era in New York City. AIDS Care 2007;19:264-272.

13. Narain JP, Lo YR. Epidemiology of HIV-TB in Asia. Indian J Med Res 2004;120:277-289.

14. Ledergerber B, Egger M, Opravil M, et al. Clinical progression and virological failure on highly active antiretroviral therapy in HIV-1 patients: a prospective cohort study. Swiss HIV Cohort Study. Lancet 1999;353:863-868.

15. Nagai $\mathrm{H}$. Factors for the onset of and the exacerbation of tuberculosis. 5. The infection and prognosis of tuberculosis among patients with immunodeficiency, especially HIV-infected patients. Kekkaku 1999;74:753-758.

16. Moreno S, Jarrin I, Iribarren JA, et al. Incidence and risk factors for tuberculosis in HIV-positive subjects by HAART status. Int J Tuberc Lung Dis 2008;12:1393-1400.

17. Sterling TR, Lau B, Zhang J, et al. Risk factors for tuberculosis after highly active antiretroviral therapy initiation in the United States and Canada: implications for tuberculosis screening. J Infect Dis 2011;204:893-901.

18. Seyler C, Toure S, Messou E, Bonard D, Gabillard D, Anglaret X. Risk factors for active tuberculosis after antiret- 
roviral treatment initiation in Abidjan. Am J Respir Crit Care Med 2005;172:123-127.

19. de Sanjose S, Mbisa G, Perez-Alvarez S, et al. Geographic variation in the prevalence of Kaposi sarcoma-associated herpesvirus and risk factors for transmission. J Infect Dis 2009;199:1449-1456.

20. Manosuthi W, Chaovavanich A, Tansuphaswadikul S, et al. Incidence and risk factors of major opportunistic infections after initiation of antiretroviral therapy among advanced HIV-infected patients in a resource-limited setting. J Infect 2007;55:464-469.

21. Cha HH, Lee SH, Lee DH, et al. Degree of disease progression in homeless HIV/AIDS patients during the first medical visit. Infect Chemother 2011;43:198-202.

22. Chattopadhyay A, Patton LL. Smoking as a risk factor for oral candidiasis in HIV-infected adults. J Oral Pathol Med 2013;42:302-308.

23. Chattopadhyay A, Patton LL. Risk indicators for HIV-associated jointly occurring oral candidiasis and oral hairy leukoplakia. AIDS Patient Care STDS 2007;21:825-832. 